\title{
FGF10 is differentially expressed in high-grade serous ovarian cancers.
}

Shahan Mamoor ${ }^{1}$

1shahanmamoor@gmail.com

East Islip, NY 11730

Ovarian cancer is the most lethal gynecologic cancer ${ }^{1}$. Soluble factors are an important part of the tumor microenvironment ${ }^{2}$. We sought to identify genes associated with high-grade serous ovarian cancer (HGSC) by comparing global gene expression profiles of normal ovary with that of primary tumors from women diagnosed with HGSC using published microarray data3,4. We found significant differential expression of the morphogen fibroblast growth factor 10 (FGF10) in high-grade serous ovarian tumors.

Keywords: ovarian cancer, high-grade serous ovarian cancer, HGSC, targeted therapeutics in ovarian cancer, systems biology of ovarian cancer, FGF10. 
The five-year survival rate for women diagnosed with high-grade serous ovarian cancer is between $30-40 \%$ and has not changed significantly in decades 8,9 . The development of novel targeted therapeutics to treat HGSC can be facilitated by an enhanced understanding of the transcriptional behavior of ovarian tumors relative to that of the normal ovary. We mined published microarray data 3,4 to compare global gene expression profiles between HGSC ovarian tumors and that of normal ovarian tissue. We identified the gene encoding fibroblast growth factor 10, FGF10, as among the most differentially expressed in HGSC tumors of the ovary FGF10 may be a gene of interest when prioritizing the study of target genes and pathways for the development of novel therapeutic interventions in high-grade serous ovarian cancers.

\section{Methods}

We used microarray data from datasets and GSE124766 ${ }^{3}$ and GSE1465564 for this differential gene expression analysis of high-grade serous carcinomas. The Benjamini and Hochberg method of $p$-value adjustment was used for ranking of differential expression but ran $p$-values were used for assessment of statistical significance of global differential expression Log-transformation of data was auto-detected, and the NCBI generated category of platform annotation was used. GSE1465564 was generated using Affymetrix Human Gene 1.0 ST Array with $n=3$ for normal ovarian tissue and $n=40$ for tumors from patients with high-grade serous ovarian cancer. GSE124766³ was generated using Agilent-014850 Whole Human Genome Microarray $4 \times 44 K$ G $4112 F$ with $n=3$ of for normal ovarian tissue and $n=8$ for tumors from patients with high-grade serous ovarian cancer. GEO2R provides mRNA expression levels only for the top 250 most differentially expressed genes.

A statistical test was performed to evaluate whether FGF10 expression was significantly different when comparing normal ovarian tissue from control subjects and primary tumors from women diagnosed with HGSC using a two-tailed, unpaired t-test with Welch's correction. Only 
p-values less than 0.05 were considered statistically significant. We used PRISM for al statistical analyses (Version 8.4.0)(455).

\section{Results}

We mined published microarray data ${ }^{3,4}$ to identify differentially expressed genes in highgrade serous ovarian cancer (HGSC), the type of ovarian cancer responsible for $70-80 \%$ of deaths resulting from the most lethal gynecologic malignancy.

FGF10 is differentially expressed in ovarian tumors from women diagnosed with HGSC.

We identified FGF10 as among the genes whose expression was most different when comparing primary HGSC tumors to normal ovarian tissue ${ }^{2}$ (Table 1). When sorting all of the transcripts measured by microarray based on change in expression between HGSC and the normal ovary, FGF10 ranked 23 out of 29088 total transcripts (Table 1). Differential expression of FGF10 in HGSC tumors was statistically significant (Table $1 ; p=9.01 \mathrm{E}-13$ ).

We analyzed a second microarray dataset ${ }^{4}$ generated using normal ovarian tissues and tumors from women diagnosed with HGSC to determine whether differential expression of FGF10 could be observed in tumors from a different group of patients. We again found that FGF10 was differentially expressed in primary HGSC tumors (Table 2). When sorting all of the transcripts measured by microarray based on change in expression between HGSC and the normal ovary, in this data set, FGF10 ranked 3909 out of 41093 total transcripts (Table 2). This means the magnitude of change in FGF10 was greater than $90.5 \%$ of the measured transcriptome. Differential expression of FGF10 in HGSC tumors in this second microarray dataset was statistically significant (Table $2 ; \mathrm{p}=8.4 \mathrm{E}-03$ ).

FGF10 is expressed at significantly lower levels in HGSC when compared to the normal ovary. We then obtained exact mRNA expression levels for the differentially expressed FGF10 transcript from both normal ovarian tissue and from high-grade serous ovarian tumors. FGF10 was expressed at significantly lower levels in high-grade serous ovarian cancers when 3 OF 11 
compared to the normal ovary (Figure $1 ; p<0.0001$ ). We calculated a mean fold change of $0.1346 \pm 0.0788$ in FGF10 expression when comparing HGSC tumors to normal ovarian tissue (Table 1).

Thus, we found using published microarray data that FGF10 was among the genes most differentially expressed in tumors from women with high-grade serous carcinomas and that FGF10 expression was significantly lower in HGSC tumors when compared to the normal ovary.

\section{Discussion}

Transcriptional profiling of HGSC in a Cancer Genome Atlas integrated genomic analysis classified HGSC into four subtypes based on gene clustering: immunoreactive, differentiated proliferative, and mesenchymal7. We sought to continue to describe the transcriptional landscape of high-grade serous ovarian cancers ${ }^{7}$ and identify genes whose differential expression was associated with HGSC by using published microarray data from primary tumors of women diagnosed with HGSC compared to transcriptome data from normal ovarian tissue 2,3 In both datasets analyzed, FGF10 was among the genes whose expression changed most significantly when comparing the normal ovary to primary HGSC tumors

Fgf10 is important for the development of the inner ear ${ }^{8}$, otic placode ${ }^{9}$ (a portion of the embryonic ectoderm from which the ear develops), prostate ${ }^{10}$, lung ${ }^{11,12}$, limb ${ }^{12}$, pancreas ${ }^{13}$ stomach $^{14}$, external genitalia ${ }^{15}$, and the cecum ${ }^{16}$. Fgf10 is expressed in human ovaries ${ }^{17}$. In the buffalo ovary, Fgf10 was specifically expressed in the granulosa cell and theca interna ${ }^{18}$. Major phenotypes displayed by Fgf10-deficient mice include cleft palate ${ }^{19}$ and congenital heart defects $^{20}$. Fgf10-deficient embryos do not possess pulmonary veins or arteries, and Fgf10 is required for correct heart positioning in the thoracic cavity20. In the brain, Fgf10-deficient mice manifest delays in differentiation of radial glia in the rostral cortex ${ }^{21}$. 
Transgenic mice in which FGF10 was conditionally expressed in the fetal lung under the promoters of surfactant protein or Clara cell secretory protein have disrupted branching morphogenesis and adenomatous malformations, and respiratory failure at birth ${ }^{22}$. FGF10 expression in the lungs after birth lead to the development of benign multifocal pulmonary tumors 22 .

Fgf10 has been labeled as an oncogene, activated by insertional mutagenesis mediated by MMTV in mouse mammary tumors ${ }^{23}$. One study documented a role for Fgf10 in cancer of the pancreas, demonstrating expression of Fgf10 from the stroma with expression of the Fgf10receptor Fgfr2 in pancreatic cancer cells ${ }^{24}$. In pancreatic cancer cell lines with FGFR2-IIIb expression, Fgf10 was able to induce invasion and migration in vitro; this was concomitant with induction of TGF-beta and MT1-MMP in CFPAC-1 cells ${ }^{24}$. This suggested that soluble signals from the stroma, including Fgf10, were important for programming of invasive and migratory properties in pancreatic cancer cells ${ }^{24}$. In PTEN-induced models of squamous cell carcinoma PTEN loss caused significant induction of Fgf1025. Ectopic expression of Fgf10 in the absence of PTEN deletion was sufficient to cause formation of skin papillomas ${ }^{25}$. There is limited information regarding fibroblast growth factor 10 or its receptors specifically in ovarian cancer One study documented mutation of FGFR2 in $2.4 \%$ of serous ovarian cancers (1 out of 41 tumors studied $)^{26}$.

Differential expression of FGF10 in HGSC should be validated in larger and separate cohorts of women with HGSC. If validated, mouse models of high-grade serous ovarian cancers $^{27-29}$ can be utilized to assess the effect of conditional expression of FGF10 in HGSO tumors post-development and on tumor cell proliferation and cancer progression in vivo. 


\section{References}

1. Guppy, A.E., Nathan, P.D. and Rustin, G.J., 2005. Epithelial ovarian cancer: a review of current management. Clinical Oncology, 17(6), pp.399-411.

2. Kenny, P.A., Lee, G.Y. and Bissell, M.J., 2007. Targeting the tumor microenvironment. Frontiers in bioscience: a journal and virtual library, 12, p.3468.

3. Hoffmann, K., Berger, H., Kulbe, H., Thillainadarasan, S., Mollenkopf, H.J., Zemojtel, T., Taube, E., Darb-Esfahani, S., Mangler, M., Sehouli, J. and Chekerov, R., 2020. Stable expansion of high-grade serous ovarian cancer organoids requires a low-Wnt environment The EMBO journal, 39(6), p.e104013.

4. Zhang, W., Klinkebiel, D., Barger, C.J., Pandey, S., Guda, C., Miller, A., Akers, S.N., Odunsi, K. and Karpf, A.R., 2020. Global DNA hypomethylation in epithelial ovarian cancer: passive demethylation and association with genomic instability. Cancers, 12(3), p.764.

5. Bowtell, D.D., Böhm, S., Ahmed, A.A., Aspuria, P.J., Bast Jr, R.C., Beral, V., Berek, J.S., Birrer, M.J., Blagden, S., Bookman, M.A. and Brenton, J.D., 2015. Rethinking ovarian cancer II: reducing mortality from high-grade serous ovarian cancer. Nature reviews Cancer, 15(11), pp.668-679.

6. Vaughan, S., Coward, J.I., Bast, R.C., Berchuck, A., Berek, J.S., Brenton, J.D., Coukos, G., Crum, C.C., Drapkin, R., Etemadmoghadam, D. and Friedlander, M., 2011. Rethinking ovarian cancer: recommendations for improving outcomes. Nature Reviews Cancer, 11(10), pp.719-725.

7. Cancer Genome Atlas Research Network, 2011. Integrated genomic analyses of ovarian carcinoma. Nature, 474(7353), p.609.

8. Alvarez, Y., Alonso, M.T., Vendrell, V., Zelarayan, L.C., Chamero, P., Theil, T., Bösl, M.R., Kato, S., Maconochie, M., Riethmacher, D. and Schimmang, T., 2003. Requirements for FGF3 and FGF10 during inner ear formation. Development, 130(25), pp.6329-6338.

9. Wright, T.J. and Mansour, S.L., 2003. Fgf3 and Fgf10 are required for mouse otic placode induction. Development, 130(15), pp.3379-3390.

10. Thomson, A.A. and Cunha, G.R., 1999. Prostatic growth and development are regulated by FGF10. Development, 126(16), pp.3693-3701

11. Li, C., Hu, L., Xiao, J., Chen, H., Li, J.T., Bellusci, S., Delanghe, S. and Minoo, P., 2005. Wnt5a regulates Shh and Fgf10 signaling during lung development. Developmental biology, 287(1), pp.86-97. 
12. Sekine, K., Ohuchi, H., Fujiwara, M., Yamasaki, M., Yoshizawa, T., Sato, T., Yagishita, N., Matsui, D., Koga, Y., Itoh, N. and Kato, S., 1999. Fgf10 is essential for limb and lung formation. Nature genetics, 21(1), pp.138-141.

13. Bhushan, A., Itoh, N., Kato, S., Thiery, J.P., Czernichow, P., Bellusci, S. and Scharfmann, R. 2001. Fgf10 is essential for maintaining the proliferative capacity of epithelial progenitor cells during early pancreatic organogenesis. Development, 128(24), pp.5109-5117.

14. Nyeng, P., Norgaard, G.A., Kobberup, S. and Jensen, J., 2007. FGF10 signaling controls stomach morphogenesis. Developmental biology, 303(1), pp.295-310.

15. Ching, S.T., Infante, C.R., Du, W., Sharir, A., Park, S., Menke, D.B. and Klein, O.D., 2018. Isl1 mediates mesenchymal expansion in the developing external genitalia via regulation of Bmp4, Fgf10 and Wnt5a. Human molecular genetics, 27(1), pp.107-119.

16. Al Alam, D., Sala, F.G., Baptista, S., Galzote, R., Danopoulos, S., Tiozzo, C., Gage, P., Grikscheit, T., Warburton, D., Frey, M.R. and Bellusci, S., 2012. FGF9-Pitx2-FGF10 signaling controls cecal formation in mice. Developmental biology, 369(2), pp.340-348.

17. Oron, G., Fisch, B., Zhang, X.Y., Gabbay-Benziv, R., Kessler-Icekson, G., Krissi, H., BenHaroush, A., Ao, A. and Abir, R., 2012. Fibroblast growth factor 10 in human ovaries. Reproductive biomedicine online, 25(4), pp.396-401.

18. Mishra, S.R., Bharati, J., Bharti, M.K., Singh, G. and Sarkar, M., 2015. Expression and localization of fibroblast growth factor 10 (FGF10) in ovarian follicle during different stages development in buffalo. Asian Journal of Animal and Veterinary Advances, 10(8), pp.433-442.

19. Rice, R., Spencer-Dene, B., Connor, E.C., Gritli-Linde, A., McMahon, A.P., Dickson, C. Thesleff, I. and Rice, D.P., 2004. Disruption of Fgf10/Fgfr2b-coordinated epithelialmesenchymal interactions causes cleft palate. The Journal of clinical investigation, 113(12), pp.1692-1700.

20. Marguerie, A., Bajolle, F., Zaffran, S., Brown, N.A., Dickson, C., Buckingham, M.E. and Kelly, R.G., 2006. Congenital heart defects in Fgfr2-IIlb and Fgf10 mutant mice. Cardiovascular research, 71(1), pp.50-60.

21. Sahara, S. and O'Leary, D.D., 2009. Fgf10 regulates transition period of cortical stem cell differentiation to radial glia controlling generation of neurons and basal progenitors. Neuron, 63(1), pp.48-62.

22. Clark, J.C., Tichelaar, J.W., Wert, S.E., Itoh, N., Perl, A.K.T., Stahlman, M.T. and Whitsett, J.A., 2001. FGF-10 disrupts lung morphogenesis and causes pulmonary adenomas in vivo. American Journal of Physiology-Lung Cellular and Molecular Physiology, 280(4), pp.L705-L715. 
23. Theodorou, V., Boer, M., Weigelt, B., Jonkers, J., Van Der Valk, M. and Hilkens, J., 2004 Fgf10 is an oncogene activated by MMTV insertional mutagenesis in mouse mammary tumors and overexpressed in a subset of human breast carcinomas. Oncogene, 23(36), pp. 6047-6055.

24. Hertzler-Schaefer, K., Mathew, G., Somani, A.K., Tholpady, S., Kadakia, M.P., Chen, Y. Spandau, D.F. and Zhang, X., 2014. Pten loss induces autocrine FGF signaling to promote skin tumorigenesis. Cell reports, 6(5), pp.818-826.

25. Nomura, S., Yoshitomi, H., Takano, S., Shida, T., Kobayashi, S., Ohtsuka, M., Kimura, F. Shimizu, H., Yoshidome, H., Kato, A. and Miyazaki, M., 2008. FGF10/FGFR2 signal induces cell migration and invasion in pancreatic cancer. British journal of cancer, 99(2), pp.305-313.

26. Byron, S.A., Gartside, M.G., Wellens, C.L., Goodfellow, P.J., Birrer, M.J., Campbell, I.G. and Pollock, P.M., 2010. FGFR2 mutations are rare across histologic subtypes of ovarian cancer. Gynecologic oncology, 117(1), pp.125-129.

27. Harrington, B.S., He, Y., Davies, C.M., Wallace, S.J., Adams, M.N., Beaven, E.A., Roche D.K., Kennedy, C., Chetty, N.P., Crandon, A.J. and Flatley, C., 2016. Cell line and patientderived xenograft models reveal elevated CDCP1 as a target in high-grade serous ovarian cancer. British journal of cancer, 114(4), pp.417-426.

28. Perets, R., Wyant, G.A., Muto, K.W., Bijron, J.G., Poole, B.B., Chin, K.T., Chen, J.Y.H. Ohman, A.W., Stepule, C.D., Kwak, S. and Karst, A.M., 2013. Transformation of the fallopian tube secretory epithelium leads to high-grade serous ovarian cancer in Brca; Tp53; Pten models. Cancer cell, 24(6), pp.751-765.

29. Kim, J., Coffey, D.M., Creighton, C.J., Yu, Z., Hawkins, S.M. and Matzuk, M.M., 2012. Highgrade serous ovarian cancer arises from fallopian tube in a mouse model. Proceedings of the National Academy of Sciences, 109(10), pp.3921-3926. 

patients with high-grade serous ovarian carcinomas.

The rank of differential expression, the probe/transcript ID, the $p$-value with respect to global differential expression, $t$, a moderated t-statistic, $B$, the log-odds of differential expression between the two groups compared, the fold change of FGF10 in the patient population when compared to control subjects, the gene and gene name are listed in this chart. 


\section{FGF10}

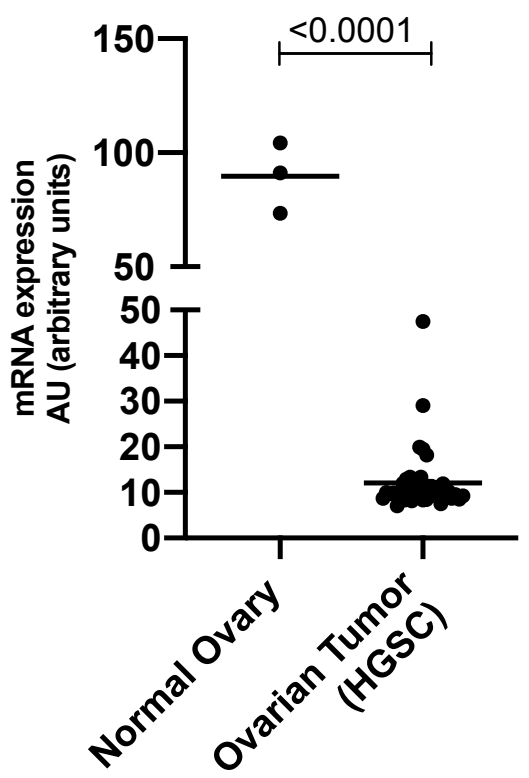

Figure 1: FGF10 is expressed at significantly lower levels in HGSC tumors when compared to the normal ovary.

The mRNA expression of FGF10 in normal ovarian tissues from control subjects (left) and in the primary tumors of patients with HGSC (right) is represented with mean mRNA expression level marked and the result of a statistical test evaluating the significance of difference in FGF10 expression between the ovary of control subjects and primary tumors from patients with HGSC, a p-value, listed above. 